\title{
Tumor Odontogénico Queratoquístico: Revisión de la Literatura a Propósito de un Caso Clínico
}

\author{
Keratocystic Odontogenic Tumor: A Review of the \\ Literature in Reference to a Clinical Case
}

Ramón Torrealba P.; Fernando Bozan S.* \& Cristina Mebus H.**

TORREALBA, P. R.; BOZAN, S. F. \& MEBUS, H. C. Tumor odontogénico queratoquístico: Revisión de la literatura a propósito de un caso clínico. Int. J. Odontostomat., 7(3):373-377, 2013.

RESUMEN: El Tumor odontogénico queratoquístico es una entidad benigna de prevalencia relativamente alta que surge desde los remanentes de la lámina dental, el cual tiene un potencial comportamiento agresivo y alta recurrencia. Este tiende a crecer lentamente dentro del canal medular en sentido anteroposterior transformándose en una gran lesión sin causar una expansión obvia. Esta revisión describe la clínica, imagenología y tratamientos actuales del Tumor Odontogénico Queratoquístico a propósito de un paciente de sexo masculino 30 años diagnosticado con esta entidad.

PALABRAS CLAVE: tumor odontogénico queratoquístico, comportamiento agresivo, recidiva.

\section{INTRODUCCIÓN}

El Tumor odontogénico queratoquístico (TOQ), antes conocido como Queratoquiste (QQ), es un tumor benigno con una prevalencia relativamente alta que surge desde los remanentes de la lamina dental. El año 2005, la Organización Mundial de la Salud dió esta nueva designación con el fin de expresar su naturaleza neoplásica, la que se ve reflejada en su potencial comportamiento agresivo y alta recurrencia.

EI TOQ representa el $4-12 \%$ de los quistes odontogénico (Cadena et al., 2010; Grasmuck \& Nelson, 2010; Shimamoto et al., 2011). Según análisis recientes, la reclasificación de $\mathrm{QQ}$ hacia TOQ lo transformaría en el tumor odontogénico mas frecuente (Kaczmarzyk et al., 2012; Servato et al., 2012). Tiene un amplio rango etario de prevalencia, siendo su peak entre la $2^{\mathrm{a}}$ y $3^{\mathrm{a}}$ década de vida, y afecta preferentemente a caucásicos de sexo masculino (Buckley et al., 2012; Cadena et al.; Grasmuck \& Nelson; Liu et al., 2012; Shimamoto et al.; Tonietto et al., 2011; Abdullah, 2011). La mandíbula es más comúnmente afectada, entre un 65-83\%, y el ángulo mandibular el sitio de mayor prevalencia (Cadena et al.; Grasmuck \& Nelson).
EI TOQ tiene un potencial invasivo ya que tiende a crecer lentamente dentro del canal medular del tejido óseo en sentido anteroposterior, transformándose en una gran lesión sin causar una expansión obvia (Ayoub et al., 2011; Cadena et al.; Grasmuck \& Nelson; Tonietto et al.). Los dientes adyacentes pueden verse desplazados sin observarse comúnmente rizálisis. Su comportamiento clínico agresivo se atribuye a su capacidad de perforar la cortical ósea (39$50 \%$ ) y afectar tejidos blandos adyacentes (Cadena et al.; Grasmuck \& Nelson; Tonietto et al.). Ocasionalmente el paciente puede presentar dolor, aumento de volumen y drenaje purulento lo que anuncia una infección secundaria de la lesión (Cadena et al.; Grasmuck \& Nelson).

Múltiples estrategias de tratamiento son todavía propuestas y controversiales (Abdullah; Grasmuck \& Nelson; Kaczmarzyk et al.). El desafío yace en disminuir el riesgo de recurrencia y morbilidad ante una extensa resección. Se describe un caso de TOQ extenso y el tratamiento médico quirúrgico utilizado para su remoción.

\footnotetext{
* Servicio de Cirugía Máxilo Facial, Hospital de Carabineros General Humberto Arriagada V., Santiago, Chile.

** Meritante Cirugía Máxilo Facial, Facultad de Odontología, Universidad Mayor, Santiago, Chile.
} 


\section{Caso Clínico}

Paciente de sexo masculino de 30 años, sin antecedentes mórbidos relevantes, hace 12 años se realizó biopsia y exéresis in toto de lesión mandibular cuyo diagnóstico fue TOQ, dicho lecho fue llenado con xenoinjerto Bio-oss. Actualmente acude por sintomatología dolorosa moderada y salida de líquido. Clínicamente puede observarse comunicación de lecho cavitario con el medio oral y salida de líquido amarillento. En la tomografía puede apreciarse una lesión radiolúcida multilocular extensa de cuerpo y rama mandibular derecha que se inicia desde el foramen mental hasta la base del proceso coronoides y cuello del cóndilo mandibular abarcándolos todo su espesor (Fig. 1). Se realizó nueva biopsia incisional de la lesión dado el tamaño de esta confirmando el diagnóstico de TOQ. Se instalaron inmediatamente 2 cánulas de descompresión y se indicó aseo con clorhexidina $0,12 \% 3$ veces al día y seguimiento radiográfico cada 6-7 meses.
Se realizaron controles tomográficos post instalación de cánula de descompresión a los 4, 10 y 17 meses (Figs. 2, 3 y 4). En dichos controles se pudo observar gran cantidad de aposición ósea lo que significó al momento de la exéresis una menor morbilidad para el paciente.

Bajo anestesia general se realizó la exéresis, curetaje mecánico y químico con solución de Carnoy de la lesión tumoral, la cual se mantendrá bajo controles periódicos debido la posibilidad de recidiva.

Tradicionalmente 6-12 meses post descompresión es considerado un periodo en el cual hay suficiente formación ósea para realizar la enucleación (Tonietto et al.; Zhao et al., 2011). Ya a los 3 meses existe una disminución del volumen de la lesión en un $55 \%$ incrementando la densidad ósea en un $46 \%$ (Zhao et al.). Pero debido a la gran extensión de la lesión se decidió esperar mayor cantidad de tiempo para disminuir la morbilidad y posibles complicaciones.

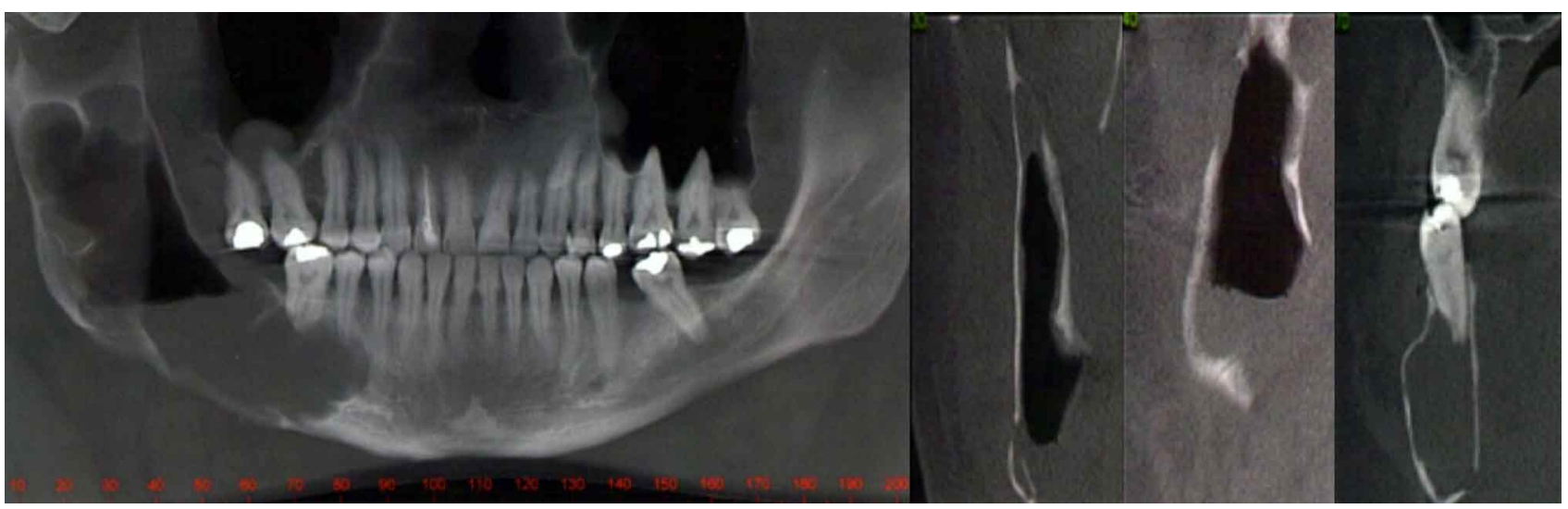

Fig. 1. En la imagen tomográfica inicial se observa la extensión y amplitud de la lesión tumoral a nivel de la rama y cuerpo mandibular derecho.

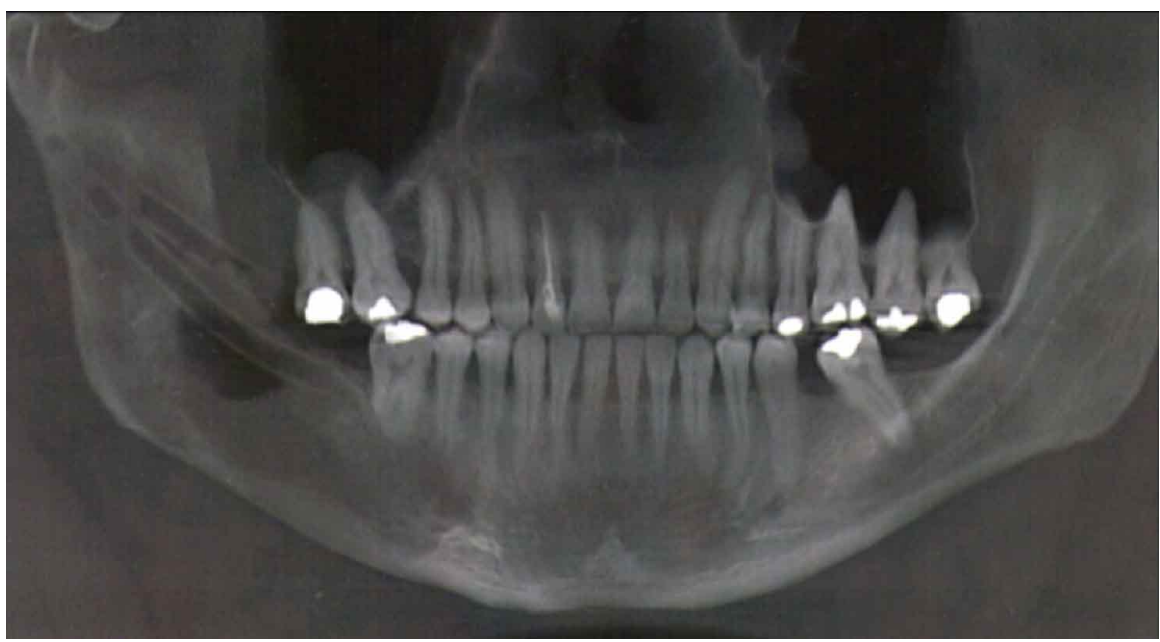

Fig. 2. Cuatro meses post instalación de cánula de descompresión ya existe aposición y cambio en la densidad ósea a nivel del ángulo y rama mandibular derecha. 


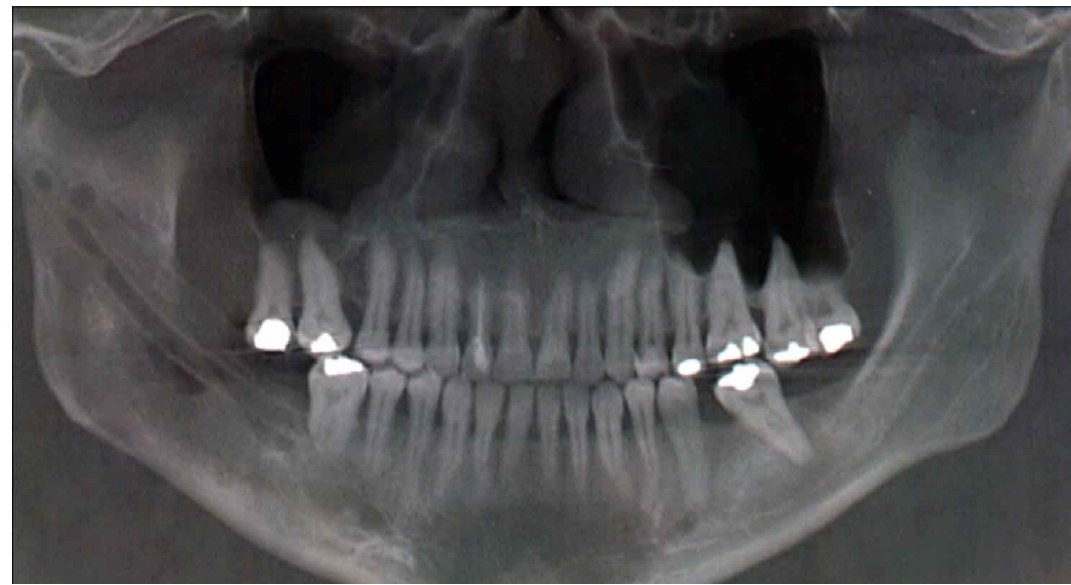

Fig. 3. Diez meses de instalado el tratamiento descompresivo.

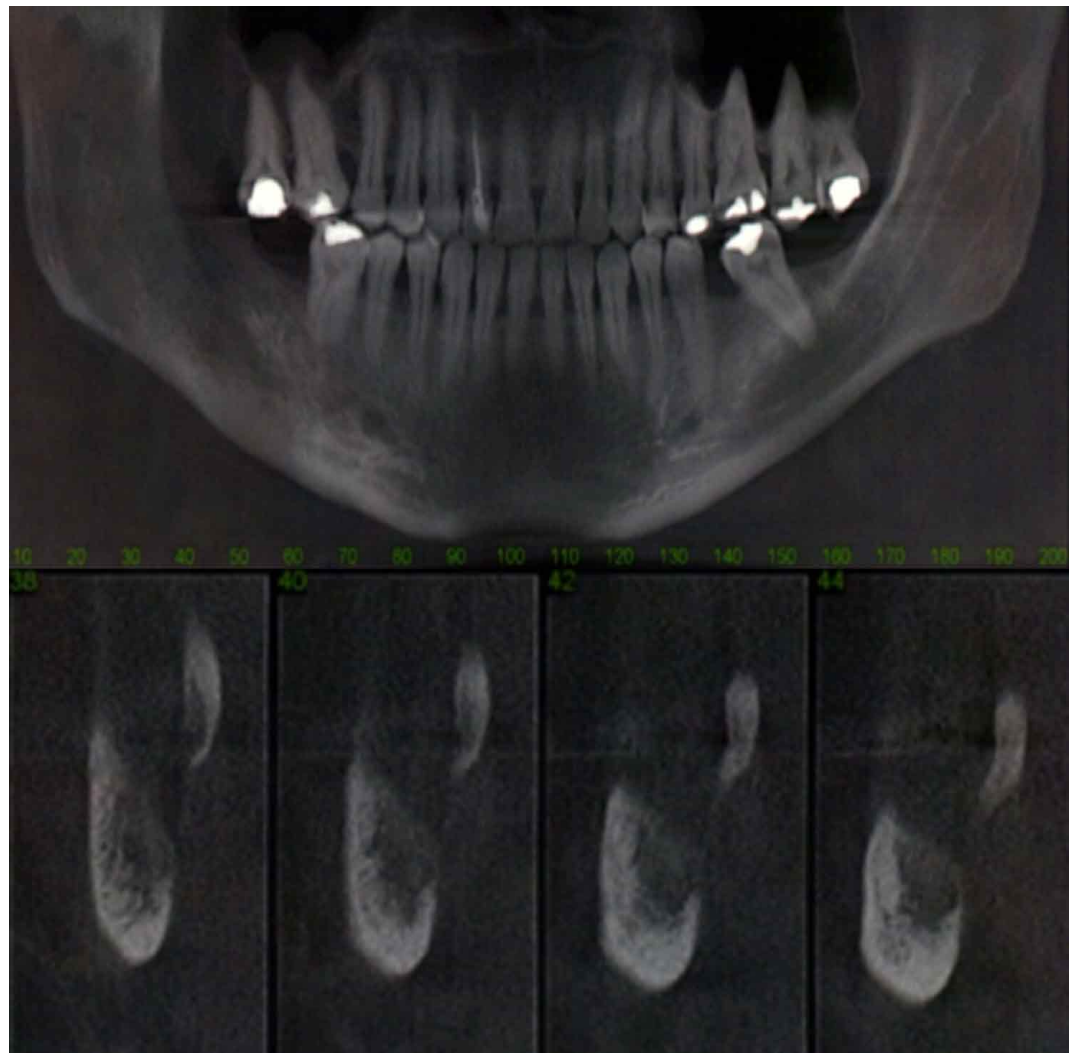

Fig. 4. Al Control de 17 meses la aposición ósea fue casi completa, solamente se aprecia una imagen radiolúcida en el trayecto de la cánula, tejido que fue resecado posteriormente.

\section{DISCUSIÓN}

El tumor odontogénico queratoquístico es una entidad benigna con una prevalencia relativamente alta que surge desde los remanentes de la lamina dental, el cual tiene un potencial comportamiento agresivo y alta recurrencia (Ayoub et al.; Cadena et al.; Grasmuck \& Nelson; Iwai et al., 2011; Kaczmarzyk et al.; Liu et al.; Shudou et al., 2012; Shuster et al., 2012).

Múltiples estudios han demostrado que el TOQ tiene un potencial invasivo, ya que este tiende a crecer lentamente dentro del canal medular del tejido óseo en sentido anteroposterior transformándose en una gran lesión sin causar una expansión obvia (Ayoub et al.; Cadena et al.; Grasmuck \& Nelson). Se han reportado casos a nivel periférico que involucran la encía, mucosa alveolar y músculo masétero, los que implican un alto porcentaje de recidiva (Cadena et al.; Grasmuck \& Nelson; Liu et al.). TOQs originados en el maxilar de características agresivas pueden extenderse hacia la órbita, fosa temporal e incluso base de cráneo (Liu et al.; Tonietto et al.).

En porcentajes bajos el epitelio del TOQ puede transformarse en un ameloblastoma y en casos muy raros en carcinoma espinocelular (Cadena et al.). A menudo es asociado con dientes retenidos (25-40\%), cuyo diagnostico diferencial es con lesiones como quiste dentígero, ameloblastoma y mixoma (Cadena et al.; Grasmuck \& Nelson). Además pueden verse características similares con granuloma central de células gigantes, tumor pardo del hiperparatiroidismo, malformaciones vasculares, querubismo, tumores óseos benignos y mieloma múltiple (Cadena et al.).

Un aspecto que debería ser destacado a cerca del TOQ es que puede representar un componente característico del Sindrome Nevoide Baso Celular, el cual está asociado en un $7 \%$ a este síndrome presentándose mayoritariamente en el sexo femenino (Grasmuck \& Nelson). Este presenta múltiples TOQ en los maxilares, carcinomas de células basales, espina bífida y calcificación de la hoz del cerebro. 
Comúnmente se hace evidente mediante hallazgos radiográficos en evaluaciones o controles de rutina con radiografías panorámicas (Grasmuck \& Nelson). Al estudio imagenológico se observa generalmente un área radiolúcida unilocular o multilocular de bordes bien delimitados y condensados ubicados habitualmente en la zona de ángulo mandibular y rama ascendente (Boffano et al., 2010; Buckley et al.; Kaczmarzyk et al.; Liu et al.; Shimamoto et al.). También podrían observarse márgenes difusos en casos de infección. La Tomografía computarizada y resonancia nuclear con contraste pueden ser utilizadas para valorar la perforación de la cortical ósea o una posible evolución en tejidos blandos (Grasmuck \& Nelson).

Estrategias de tratamiento son todavía propuestas y controversiales. El desafío yace en disminuir el riesgo de recurrencia y morbilidad ante una extensa resección (Ayoub et al.; Grasmuck \& Nelson; Kaczmarzyk et al.). Las causas de recurrencia son esencialmente la remoción incompleta de la membrana quística y el crecimiento desde pequeños quistes satélites o nidos de epitelio odontogénico dejados después de la enucleación (Iwai et al.; Liu et al.; Shudou et al.; Tonietto et al.; Zhou et al.). Numerosas modalidades de tratamientos han sido empleadas en el manejo del TOQ tanto conservador como radical. Dentro del tratamiento conservador podemos encontrar: marsupialización o descompresión, enucleación simple, enucleación con curetaje periférico (mecánico o químico), descompresión seguida de enucleación. El tratamiento quirúrgico radical es más agresivo, el cual puede comprender resección marginal, resección en bloque y hemimaxilectomía o hemimandibulectomía (Kaczmarzyk et al.; Tonietto et al.). El uso de coadyuvantes o curetaje químico como crioterapia con nitrógeno líquido o solución de Carnoy han sido recomendados para eliminar los posibles quistes satélites en los limites óseos (Cadena et al.; Grasmuck \& Nelson). El más utilizado es la solución de Carnoy, la cual penetra entre las trabéculas óseas, desvitalizando y fijando las células tumorales (Kaczmarzyk et al.).
Según estudios, se ha reportado en la literatura una recurrencia entre un $25-56 \%$ (Ayoub et al.; Buckley et al.; Cadena et al.; Grasmuck \& Nelson; Kaczmarzyk et al.), las cuales se ven mayormente en los 5 primeros años postquirúrgicos (Liu et al.; Tonietto et al.). El tratamiento de enucleación simple tiene una tasa de recurrencia entre $17-56 \%$, pero si como complemento aplicamos solución de Carnoy o descompresión previa a la enucleación disminuye entre 1-8,7\% (Grasmuck \& Nelson; Kaczmarzyk et al.; Liu et al.; Zhao et al.; Zhou et al.). Finalmente la resección es reportada como un tratamiento esencialmente sin recurrencias, pero puede ser inaceptable dada la naturaleza benigna de la enfermedad además de las numerosas complicaciones asociadas como deformación facial, perdidas dentarias, infecciones de injerto trasplantado y alteraciones sensitivas por daño del nervio alveolar inferior (Boffano et al.; Grasmuck \& Nelson; Kaczmarzyk et al.; Shudou et al.).

Se han propuesto pautas de tratamiento dependientes de factores citados anteriormente como (Cadena et al.; Kaczmarzyk et al.):

i. En pacientes con lesiones de menor tamaño es conveniente el manejo mediante enucleación de la lesión, curetaje periférico y aplicación de soluciones adyuvantes en las paredes cavitarias (Iwai et al.)

ii. En lesiones de mayores dimensiones se propone la descompresión de esta previa a la enucleación, ya que disminuye el tamaño del tumor, la extensión de la cirugía y su morbilidad (Boffano et al.; Grasmuck \& Nelson; Kaczmarzyk et al.; Tonietto et al.).

iii. Lesiones cuya extensión producen una ruptura de las corticales óseas y se vean afectados tejidos blandos debiesen ser manejadas con una terapéutica radical, ya que es considerada como una lesión agresiva lo que conlleva un grado de recurrencia mayor (Boffano et al.; Liu et al.).

TORREALBA, P. R.; BOZAN, S. F. \& MEBUS, H. C. Keratocystic odontogenic tumor: A review of the literature in reference to a clinical case. Int. J. Odontostomat., 7(3):373-377, 2013.

ABSTRACT: Keratocystic Odontogenic tumor is a benign entity with relatively high prevalence that arises from remains of dental lamina. It has a potentially aggressive behaviour, high recurrence and anteroposterior slow growth in the medullar canal, which can become large lesion without obvious expansion. This review describes clinical, imagenological and current treatments of Keratocystic Odontogenic Tumor in 30-year-old male patient diagnosed with this entity.

KEY WORDS: keratocystic odontogenic tumor, aggressive behaviour, recurrence. 


\section{REFERENCIAS BIBLIOGRÁFICAS}

Abdullah, W. A. Surgical treatment of keratocystic odontogenic tumour: A review article. Saudi Dent. J., 23(2):61-5, 2011.

Ayoub, M. S.; Baghdadi, H. M. \& El-Kholy, M. Immunohistochemical detection of laminin-1 and Ki-67 in radicular cysts and keratocystic odontogenic tumors. BMC Clin. Pathol., 11:4, 2011.

Boffano, P.; Ruga, E. \& Gallesio, C. Keratocystic odontogenic tumor (odontogenic keratocyst): preliminary retrospective review of epidemiologic, clinical, and radiologic features of 261 lesions from University of Turin. J. Oral Maxillofac. Surg., 68(12):2994-9, 2010.

Buckley, P. C.; Seldin, E. B.; Dodson, T. B. \& August, M. Multilocularity as a radiographic marker of the keratocystic odontogenic tumor. J. Oral Maxillofac. Surg., 70(2):320-4, 2012.

Cadena, A. J. L.; Muñiz, L. G.; Tapia, P. D. C.; Flores, T. A. \& Orozco, J. M. E. Queratoquiste odontogénico. Revisión de 20 años en la Unidad Médica de Alta Especialidad, Hospital de Especialidades CMN «La Raza» IMSS 19802000. Rev. Mex. Cir. Bucal Maxilofac., 6(1):4-13, 2010.

Grasmuck, E. A. \& Nelson, B. L. Keratocystic odontogenic tumor. Head Neck Pathol., 4(1):94-6, 2010.

Iwai, T.; Hirota, M.; Maegawa, J. \& Tohnai, I. Use of methylene blue for precise peripheral ostectomy of keratocystic odontogenic tumour. Br. J. Oral Maxillofac. Surg., 49(8):e84-5, 2011.

Kaczmarzyk, T.; Mojsa, I. \& Stypulkowska, J. A systematic review of the recurrence rate for keratocystic odontogenic tumour in relation to treatment modalities. Int. J. Oral Maxillofac. Surg., 41(6):756-67, 2012.

Liu, B.; Cai, Y.; Wang, S. P. \& Zhao, Y. F. Recurrent keratocystic odontogenic tumor in the masseter muscle overlying the boney perforations: a case report. Oral Surg. Oral Med. Oral Pathol. Oral Radiol., 113(4):e1-5, 2012.

Servato, J. P.; de Souza, P. E.; Horta, M. C.; Ribeiro, D. C.; de Aguiar, M. C.; de Faria, P. R.; Cardoso, S. V. \& Loyola, A. M. Odontogenic tumours in children and adolescents: a collaborative study of 431 cases. Int. J. Oral Maxillofac. Surg., 41(6):768-73, 2012.

Shimamoto, H.; Kishino, M.; Okura, M.; Chindasombatjaroen, J.; Kakimoto, N.; Murakami, S. \& Furukawa, S. Radiographic features of a patient with both cementoossifying fibroma and keratocystic odontogenic tumor in the mandible: a case report and review of literature. Oral
Surg. Oral Med. Oral Pathol. Oral Radiol. Endod., 112(6):798-802, 2011.

Shudou, H.; Sasaki, M.; Yamashiro, T.; Tsunomachi, S.; Takenoshita, Y.; Kubota, Y.; Ninomiya, T.; Kawazu, T. \& Mori, Y. Marsupialisation for keratocystic odontogenic tumours in the mandible: longitudinal image analysis of tumour size using 3D visualised CT scans. Int. J. Oral Maxillofac. Surg., 41(3):290-6, 2012.

Shuster, A.; Shlomi, B.; Reiser, V. \& Kaplan, I. Solid keratocystic odontogenic tumor--report of a nonaggressive case. J. Oral Maxillofac. Surg., 70(4):86570, 2012.

Tonietto, L.; Borges, H. O.; Martins, C. A.; Silva, D. N. \& Sant'Ana Filho, M. Enucleation and liquid nitrogen cryotherapy in the treatment of keratocystic odontogenic tumors: a case series. J. Oral Maxillofac. Surg., 69(6):e112-7, 2011.

Zhao, Y.; Liu, B.; Han, Q. B.; Wang, S. P. \& Wang, Y. N. Changes in bone density and cyst volume after marsupialization of mandibular odontogenic keratocysts (keratocystic odontogenic tumors). J. Oral Maxillofac. Surg., 69(5):1361-6, 2011.

Zhou, H.; Hou, R.; Ma, Q.; Wu, K.; Ding, Y.; Qin, R. \& Hu, K. Secondary healing after removal of large keratocystic odontogenic tumor in the mandible: enucleation followed by open packing of iodoform gauze. J. Oral Maxillofac. Surg., 70(7):1523-30, 2012.

Dirección para Correspondencia:

Dr. Fernando Bozán Santibáñez

Regina Pacis 760 Dpto. 906

Código Postal: 7770066

Ñuñoa

Santiago

CHILE

Email: f.bozan@gmail.com

Recibido : 14-01-2013

Aceptado: 28-08-2013 